\title{
Eclipsing binary millisecond pulsar PSR J1740-5340 - evolutionary considerations and observational test
}

\author{
E. Ergma ${ }^{1}$ and M. J. Sarna ${ }^{2}$ \\ ${ }^{1}$ Physics Department, Tartu University, Ülikooli 18, 50090 Tartu, Estonia \\ e-mail: ene@physic.ut.ee \\ 2 N. Copernicus Astronomical Center, Polish Academy of Sciences, ul. Bartycka 18, 00-716 Warsaw, Poland \\ e-mail: sarna@camk.edu.pl
}

Received 12 March 2002 / Accepted 12 November 2002

\begin{abstract}
We perform evolutionary calculations for a binary system to produce observed binary parameters for the PSR J1740-5340. Our calculations support the model proposed by D'Amico et al. (2001) in which this binary may be the progenitor of a millisecond pulsar + helium white dwarf system. We propose an observational test to verify this hypothesis. We propose that observations of abundances of C, N, O elements in the spectra of the optical companion of PSR J 1740-5340 may give additional information about the nature of the secondary being either i) a perturbed low-mass main-sequence star or ii) an evolved star with helium core and thin hydrogen envelope which has lost its main envelope during semi-detached evolution.
\end{abstract}

Key words. stars: evolution - pulsar: individual: PSR J1740-5340

\section{Introduction}

The millisecond pulsar PSR J1740-5340 ( $\left.P_{\mathrm{pul}}=3.65 \mathrm{~ms}\right)$ was discovered during a systematic search of Galactic globular clusters using the Parkes radio telescope (D'Amico et al. 2001a,b). PSR J1740-5340 in the globular cluster NGC 6397 is a member of a binary system with a relatively wide orbit of period 1.35 days, a companion with mass $M_{\mathrm{c}}>0.19 M_{\odot}$, and at a frequency of $v=1.4 \mathrm{GHz}$ is eclipsed for about $40 \%$ of its orbit. The spin-down age of PSR J1740-5340 $\left(\tau_{\text {sd }}=P_{\text {pul }} / 2 \dot{P}_{\text {pul }}\right)$ is $\sim 350 \mathrm{Myr}$ and its surface magnetic field is $B=3.2 \times 10^{19}\left(P_{\mathrm{pul}} \dot{P}_{\mathrm{pul}}\right)^{1 / 2} \sim 8 \times 10^{8}$ G. Ferraro et al. (2001) report the optical identification of the companion to the millisecond pulsar (MSP). They found that the observed modulation is just $\sim 0.2 \mathrm{mag}$; this can be reproduced only if the companion has almost filled its Roche lobe and the orbital plane is nearly edge-on $\left(i \sim 90^{\circ}\right)$. These two requirements are met by a companion of mass in the range $0.19-0.22 M_{\odot}$, whose Roche lobe radius $\left(1.32-1.42 R_{\odot}\right)$ just matches the lower limit for the observed radius $R_{\mathrm{c}} \sim 1.3-1.8 R_{\odot}$. D'Amico et al. (2001a,b) and Ferraro et al. (2001) propose two hypotheses for the origin of this system.

To explain the large mass loss rate they proposed that the companion is an evolved star that spun up the pulsar to its millisecond period. The mass accretion and spinning up of the pulsar would now be inhibited by the pulsar wind flux which, could expel the matter overflowing from the Roche lobe of the

Send offprint requests to: M. J. Sarna, e-mail: sarna@camk.edu.pl companion. This would be the first confirmed example of a recently born MSP.

Another hypothesis is that the optical companion is a MainSequence (M-S) star perturbed by the energetic flux emitted from the millisecond pulsar. Only 5\% of the impinging power would be needed to sustain the inferred mass loss rate of $\dot{M}<2 \times 10^{-11} M_{\odot} \mathrm{yr}^{-1}$ (D'Amico et al. 2001b). This model should be more easily applicable to a M-S star, because a large convective envelope favours bloating of the star.

Grindlay et al. (2002) propose another interesting hypothesis that it appears plausible that a typical old ( $\tau>1 \mathrm{Gyr}$ ) MSP in a dense cluster core might undergo one or more MSP-LMXB transformation cycles. PSR J1740-5340 in NGC 6397 has the advantages of having been recently scattered out of a still higher density $\left(\sim 10^{6} \mathrm{pc}^{-3}\right)$ core collapsed cluster and possible having exchanged its companion (Ferraro et al. 2001; Grindlay et al. 2001). So they proposed that this system need not just "born", it may instead have just been reborn.

In this research note we shall show that it is possible to reproduce the observational parameters for the system PSR J1740-5340 and that the hypothesis proposed by D'Amico et al. (2001a) and Ferraro et al. (2001) really may work. If so, we are observing a binary right before the formation of a detached He white dwarf + millisecond pulsar system. We also propose an observational test to distinguish between the two hypotheses (M-S star vs. evolved star).

While the manuscript was reviewed, two papers were published that are relevant for our manuscript (Kałużny et al. 2003; 
Orosz \& van Kerkwijk 2003). We have not incorporated these papers in ours but conclude that they describe results consistent with our conclusion.

\section{The evolutionary code}

The evolutionary sequences we have calculated consist of two main phases:

(i) detached evolution lasting until the companion fills its Roche lobe (RLOF);

(ii) semi-detached evolution.

\subsection{Detached phase}

The duration of the detached phase is somewhat uncertain; it may be determined either by the nuclear time-scale or by the much shorter time-scale of the orbital angular momentum loss caused by a magnetized stellar wind.

\subsection{Semi-detached phase}

In our calculations we assume that the semi-detached evolution of a binary system is a combination of an accretion phase during which the neutron star will spin-up and its mass increases, and a non-conservative phase, i.e. the total mass and angular momentum of the system are not conserved (during "propeller" and "magneto-dipole radiation" phases). The formalism which we have adopted is described in Muslimov \& Sarna (1993, 1995). We introduce the parameter, $f_{1}$, characterizing the loss of mass from the binary system and defined by the relations

$\dot{M}=\dot{M}_{\mathrm{sg}} f_{1} \quad$ and $\quad \dot{M}_{\mathrm{ns}}=-\dot{M}_{\mathrm{sg}}\left(1-f_{1}\right)$,

where $\dot{M}$ is the mass-loss rate from the system, $\dot{M}_{\mathrm{sg}}$ is the rate of mass-loss from the donor (secondary) star, and $\dot{M}_{\mathrm{ns}}$ is the accretion rate onto the neutron star (primary). The matter leaving the system will carry away its intrinsic angular momentum according to the

$\frac{j}{J}=f_{1} \frac{M_{\mathrm{ns}} \dot{M}_{\mathrm{sg}}}{M_{\mathrm{sg}} M}+\frac{j}{J}{ }_{\mathrm{MSW}} \quad \mathrm{yr}^{-1}$,

where $M, M_{\mathrm{ns}}$ and $M_{\mathrm{sg}}$ are the total mass of the system, and the masses of the neutron star and donor star respectively. At the beginning of mass transfer matter will accrete onto neutron star and spin it up $\left(f_{1}=0\right)$. After accretion of $0.2-0.25 M_{\odot}$ the binary enters into a "propeller phase", which is followed by the magneto-dipole radiation phase. During these two phases the evolution is non-conservative $\left(f_{1}=1\right)$.

We also assume that the donor star, possessing a convective envelope, experiences magnetic braking (Mestel 1968; Mestel \& Spruit 1987), and as a consequence of this, the system loses orbital angular momentum at the rate

$\bar{J}_{\mathrm{MSW}}=-3 \times 10^{-7} \frac{1}{K^{2}} \frac{M^{2} R_{\mathrm{sg}}^{4}}{M_{\mathrm{sg}} M_{\mathrm{ns}} A^{5}} \quad \mathrm{yr}^{-1}$

where the value of $K \sqrt{M_{\mathrm{sg}}}$ corresponds to the parameter $\lambda$ of Verbunt \& Zwaan (1981).
The angular momentum losses that accompany the mass loss due to "propeller" and magnetic braking angular momentum loss have comparable efficiencies. For illustration we estimated the characteristic time-scales $\left[\tau=(\dot{J} / J)^{-1}\right]$ for both mechanisms. We assume a binary system at the beginning of the "propeller" stage with components of 0.6 and $1.65 M_{\odot}$ and orbital period $1.24 \mathrm{~d}$. The secondary has a radius $1.88 R_{\odot}$; $f_{1}=1$ and the mass transfer rate from the secondary to neutron star is $5 \times 10^{-9} M_{\odot} \mathrm{yr}^{-1}$. We obtain $\tau_{\mathrm{MSW}} \sim 2 \times 10^{15} \mathrm{~s}$ and $\tau_{\text {prop }} \sim 5 \times 10^{15} \mathrm{~s}$.

In the semi-detached (magneto-dipole radiation) phase we have also included the effect of illumination of the donor star by the millisecond pulsar. In our calculations we assume that the illumination of the component by the hard (X-ray and $\gamma$-ray) radiation from the pulsar leads to additional heating of its photosphere (Muslimov \& Sarna 1993). The effective temperature, $T_{\text {eff }}$, of the companion during the illumination stage is determined from the relation

$L_{\text {in }}+f_{2}\left(\frac{R_{\mathrm{sg}}}{2 a}\right)^{2} L_{\mathrm{rot}}=4 \pi \sigma R_{\mathrm{sg}}^{2} T_{\mathrm{eff}}^{4}$

where $L_{\text {in }}$ is the intrinsic luminosity corresponding to the radiation flux coming from the stellar interior $\sigma$ is the StefanBoltzmann constant and $R_{\mathrm{sg}}$ is the stellar radius, $L_{\mathrm{rot}}$ is the "rotational luminosity" of the neutron star due to magneto-dipole radiation (plus a wind of relativistic particles)

$L_{\mathrm{rot}}=\frac{2}{3 c^{3}} B^{2} R_{\mathrm{ns}}^{6}\left(\frac{2 \pi}{P_{\mathrm{pul}}}\right)^{4}$

where $R_{\mathrm{ns}}$ is the neutron star radius, $B$ is the value of the magnetic field strength of the neutron star and $P_{\text {pul }}$ is the pulsar spin period. $f_{2}$ is a factor characterizing the efficiency of transformation of irradiation flux into thermal energy (in our case we take $f_{2}=2 \times 10^{-3}$ ). Note that in our calculations the effect of irradiation is formally treated by means of modification of the outer boundary condition, according to the above relation (4).

We have not included $\mathrm{X}$-ray heating during the accretion stage - low-mass X-ray binary phase (LMXB). Spherically symmetric illumination in LMXBs can have a strong effect on the structure of low-mass secondaries, leading to a stellar expansion, which affects the mass transfer rate (Podsiadlowski 1991; Harpaz \& Rapppaport 1991; Ergma \& Fedorova 1992). In reality, the illumination will not be spherically symmetric and its effect will depend on the optical depth at which energy is deposited and how fast is it can be transported to the cool side of the star. This problem is still open.

\subsection{Pulsar spin evolution}

We also follow the rotational evolution of the neutron star, which is determined mainly by the accretion rate and the temporal evolution of the magnetic field of the neutron star. For the temporal evolution of the magnetic field the model by Urpin \& Muslimov (1992) has been used. 
As the neutron star spins up, the net accretion torque gradually decreases and the spin period reaches its equilibrium value given (see e.g. van den Heuvel 1977) by

$P_{\text {eq }}=2.1 B_{9}^{6 / 7}\left(\frac{\dot{M}_{\text {sg }}}{\dot{M}_{\text {edd }}}\right)^{-3 / 7} \mathrm{~ms}$

where $B_{9}=B / 10^{9} \mathrm{G}$ and $\dot{M}_{\text {edd }}$ is the Eddigton mass accretion rate.

The accretion phase onto a rotating neutron star is centrifugally inhibited when the Alfvén radius $\left(R_{\mathrm{A}} \sim 2.2 \times 10^{6} \mu_{26}^{4 / 7}\right.$ $\left(R_{6} L_{37}\right)^{-2 / 7} M_{\mathrm{ns}}^{1 / 7} \mathrm{~cm}$ where $R_{6}$ is the neutron star radius in $10 \mathrm{~km}, \mu_{26}$ is the neutron star magnetic moment in units of $10^{26} \mathrm{G} \mathrm{cm}^{3}$ and $L_{37}$ is the accretion luminosity in units of $10^{37} \mathrm{erg} \mathrm{s}^{-1}$ ) is larger than the corotation radius $R_{\mathrm{co}}=2.6 \times$ $10^{6} M_{\mathrm{ns}}^{1 / 3} P_{\mathrm{pul}(-3)}^{2 / 3} \mathrm{~cm}$. In this case the accreting matter could be ejected from the system - the " propeller" phase (Illarionov \& Sunyaev 1975). Recently Burderi et al. (2001) used a ShakuraSunyaev accretion disk model to determine the critical orbital period value $P_{\text {orb,crit }}$, such that, if $P_{\text {orb }}>P_{\text {orb,crit }}$ then the radio ejection phase starts in which the mechanism that drives mass overflow through L1 is still active, while the pulsar radiation pressure prevents mass accretion. According to Burderi et al. (2002) this condition is satisfied for an orbital period longer than

$$
\begin{gathered}
P_{\text {orb }, \text { crit }}=0.75 \alpha^{-54 / 25} n_{0.615}^{-12 / 5} M_{\mathrm{ns}}^{107 / 50} M^{-1 / 2} \\
g\left(M_{\mathrm{ns}}, M_{\mathrm{sg}}\right)^{-1.5} \mu_{26}^{-24 / 5} P_{\mathrm{pul}(-3)}^{48 / 5} \dot{M}_{-10}^{51 / 25} \mathrm{hr}
\end{gathered}
$$

where $\alpha$ is the Shakura-Sunyaev viscosity parameter $(=1)$, $n_{0.615}=n / 0.615 \sim 1, g\left(M_{\mathrm{ns}}, M_{\mathrm{sg}}\right)=1-0.462\left(M_{\mathrm{sg}} / M\right)^{1 / 3}$, $\dot{M}_{-10}=\dot{M}_{\mathrm{sg}} / 10^{-10} M_{\odot} / \mathrm{yr}$, and $P_{\operatorname{pul}(-3)}$ is the neutron star spin period in milliseconds.

\subsection{Nuclear network}

Our nuclear reaction network is based on that of Kudryashov \& Ergma (1980), who included the reactions of the CNO tricycle in their calculations of hydrogen and helium burning in the envelope of an accreting neutron star. We have included the reactions of the proton-proton $(\mathrm{PP})$ chain. Hence we are able to follow the evolution of the nuclei: ${ }^{1} \mathrm{H},{ }^{3} \mathrm{He},{ }^{4} \mathrm{He},{ }^{7} \mathrm{Be},{ }^{12} \mathrm{C},{ }^{13} \mathrm{C}$, ${ }^{13} \mathrm{~N},{ }^{14} \mathrm{~N},{ }^{15} \mathrm{~N},{ }^{14} \mathrm{O},{ }^{15} \mathrm{O},{ }^{16} \mathrm{O},{ }^{17} \mathrm{O}$ and ${ }^{17} \mathrm{~F}$. We use the reaction rates from: Fowler et al. (1967, 1975), Harris at al. (1983) and Pols et al. (1995).

\section{Results of calculations}

We perform calculations for the following system parameters: $M_{\mathrm{sg}, i}=0.85 M_{\odot}, M_{\mathrm{ns}, i}=1.4 M_{\odot}, P_{\mathrm{orb}, i}(\mathrm{RLOF})=1.17 \mathrm{~d} M_{\mathrm{sg}, i}=$ $0.8 M_{\odot}, M_{\mathrm{ns}, i}=1.4 M_{\odot}, P_{\mathrm{orb}, i}(\mathrm{RLOF})=1.14 \mathrm{~d}$ and chemical composition: $X=0.7, Z=0.003$. In Fig. 1 we show the massloss rate from the donor star versus secondary mass for $M_{\mathrm{sg}}=$ $0.85 M_{\odot}$ (a similar picture is found for $M_{\mathrm{sg}}=0.8 M_{\odot}$ ). The small jump in mass loss rate near $M_{\mathrm{sg}}=0.6 M_{\odot}$ is connected with transformation from the accretion phase $\left(f_{1}=0\right)$ to the non-conservative approach $\left(f_{1}=1\right)$. Just near this mass condition $R_{\mathrm{A}}>R_{\text {co }}$ is fulfilled. Near to $M_{\mathrm{sg}} \sim 0.21 M_{\odot} P_{\text {orb }}>P_{\text {orb,crit }}$ and the radio ejection phase starts.

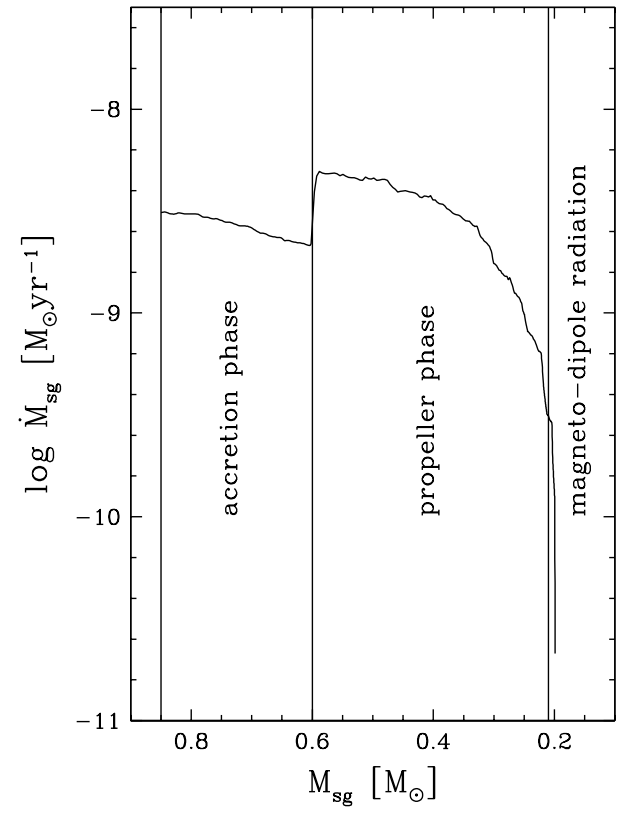

Fig. 1. The evolution of the mass transfer rate versus secondary mass. To the right of the second vertical line the pulsar is in the "propeller phase" which is followed by the "magneto-dipole radiation phase".

If (like in the case of study) Roche lobe filling of secondary occurs near the bifurcation period (the bifurcation period separates the formation of the converging systems: $P_{\mathrm{f}}<$ $P_{\text {orb }, i}(\mathrm{RLOF})$ from the diverging systems: $P_{\mathrm{f}}>P_{\text {orb }, i}(\mathrm{RLOF})$, where $P_{\mathrm{f}}$ and $P_{\mathrm{orb}, i}(\mathrm{RLOF})$ are the final and initial orbital periods, respectively) then, as was already found by Tutukov et al. (1985), the orbital evolution is quite different from the case when the secondary Roche lobe filling occurs far from bifurcation period. At the beginning, the mass loss rate is high and the pulsar will spin up its to current spin period; then the mass loss rate quickly decreases. Near the bifurcation period the nuclear time-scale of the secondary and the angular momentum loss time-scale are very close to each other. Therefore, during the binary evolution orbital period changes are insignificant in comparison with the initial orbital period (Tutukov et al. 1985; Ergma et al. 1998). In Fig. 2 we show the orbital period evolution versus pulsar spin period. During evolution the current orbital period value is achieved twice: the first time when $M_{\mathrm{sg}} \sim 0.35 M_{\odot}$ and a second time when $M_{\mathrm{sg}} \sim 0.2 M_{\odot}$.

During semi-detached evolution, near $P_{\text {orb }}=1.35$ days the secondary mass has decreased to $M_{\mathrm{sg}} \sim 0.2 M_{\odot}, M_{\mathrm{ns}}=$ $1.65 M_{\odot}$, and the total evolutionary time is $\sim 10^{10} \mathrm{yr}$ (NGC 6397 age is $11.4 \pm 1.1 \mathrm{Gyr}$ by Rakos et al. 2001), the secondary radius is $\sim 1.38 R_{\odot}$ and its effective temperature $5700 \mathrm{~K}$. Both values fit well with observed values (D'Amico et al. 2001a; Ferraro et al. 2001). From Taylor et al. (2001) we know that $m_{v}=16.66 \mathrm{mag}$ for the optical component of PSR J1740+5340. From Harris (1996) we find that the distance modulus to NGC 6397 is 12.25 mag. These numbers give an absolute magnitude $M_{v}=4.41$. From our theoretical temperature and radius determination we calculated $M_{\mathrm{bol}}=$ 4.11. The bolometric correction from Houdashelt et al. (2000) is $\mathrm{BC}=0.272 \pm 0.007$ for an assumed metallicity of the cluster 


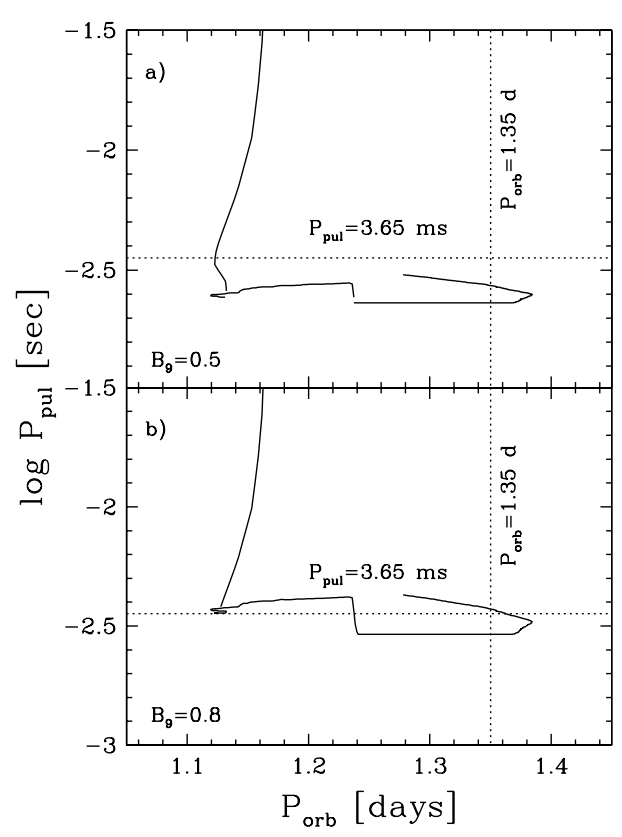

Fig. 2. The evolution of the orbital period versus pulsar spin period.

$[\mathrm{Fe} / \mathrm{H}]=-2.0$. The theoretical value of $M_{v}=4.38$ agree well with the Taylor at al. (2001) determination.

Figures $3 \mathrm{a}$ and $\mathrm{b}$ depict the temporal behaviour of the neutron star spin period, $P_{\mathrm{pul}}$ and $P_{\mathrm{eq}}$, in the cases when $B=5 \times 10^{8}$ and $8 \times 10^{8} \mathrm{G}$, respectively, at the beginning of the accretion stage.

Note also, that for binary system parameters as above, the pulsar spin period is 2.5 and $3.5 \mathrm{~ms}$ for $5 \times 10^{8}$ and $8 \times 10^{8} \mathrm{G}$, respectively.

\section{Observational test}

We propose that observations of abundances of $\mathrm{C}, \mathrm{N}, \mathrm{O}$ elements in the spectra of the optical the companion of PSR J1740-5340 may give additional information about the nature of the secondary, being either i) a perturbed low-mass $\mathrm{M}-\mathrm{S}$ star or ii) an evolved star with helium core and thin hydrogen envelope which has lost its main envelope during the accretion phase. In Fig. 4 we present the evolution of the surface chemical composition of the optical companion as a function of the secondary mass (for case ii)). Near orbital period $P_{\text {orb }}=$ $1.35 \mathrm{~d}$, for the evolved star carbon is depleted by $2.6 \mathrm{dex}$ in comparison to cosmic abundances. The abundance of oxygen will remain at the level of a metal poor star. In fact in Fig. 4 the abundance of oxygen does not change during semi-detached evolution. The abundance of oxygen is due to the assumed Population II chemical composition. Only nitrogen lines will show cosmic abundances (see Fig. 4). In the case of the lowmass $\mathrm{M}-\mathrm{S}$ star the $\mathrm{C}, \mathrm{N}, \mathrm{O}$ abundances will be as for the halo and disc metal-poor $([\mathrm{Fe} / \mathrm{H}]<-1.5)$ dwarfs (Carretta et al. 2000 and references therein). In the case of the evolved star (helium) $\mathrm{N}$ will be overabundant (approximately solar) when $\mathrm{O}$ abundance will be as for metal poor dwarfs. There will be lack of carbon lines in the evolved star spectrum.

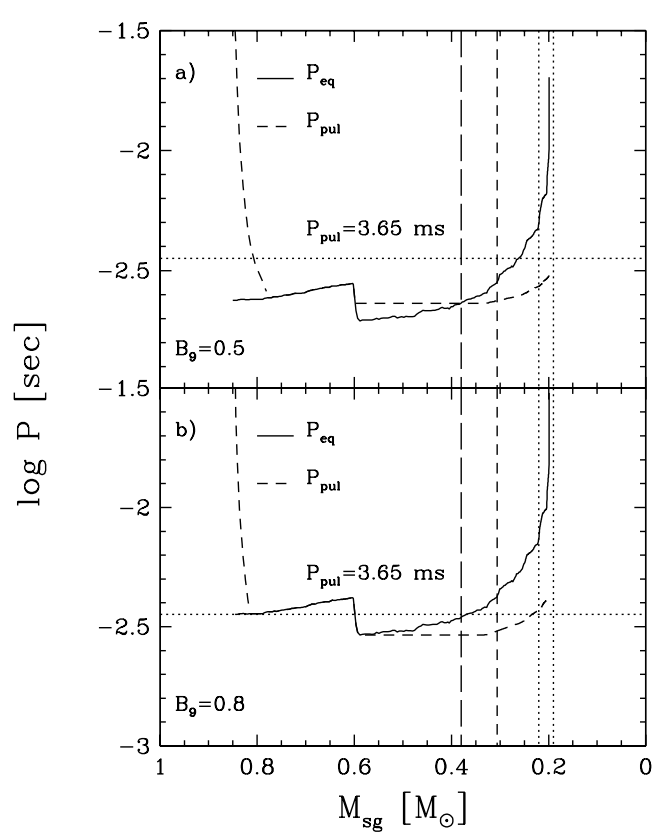

Fig. 3. The evolution of the spin period $\left(P_{\text {pul }}\right)$ of the pulsar and equilibrium period $\left(P_{\text {eq }}\right)$ versus the mass of the secondary for two values of magnetic field strength: $5 \times 10^{8} \mathrm{G}$ a) and $8 \times 10^{8} \mathrm{G} \mathrm{b}$ ). The horizontal dotted line shows the spin period of PSR J1740-53. The vertical dotted lines show the mass range of the secondary star (Ferraro et al. 2001) and the dashed and long-dashed lines are an upper limits for the secondary mass according to Kałużny et al. (2003) and Orosz \& van Kerkwijk (2003), respectively.

\section{Discussion and conclusions}

After we sent the first version of our paper to A\&A we learnt that Burderi et al. (2002) have discussed a similar evolutionary scenario for this system. Unfortunately they present their results in a very abbreviated way and it is difficult to compare our and their results.

We accept the D'Amico et al. (2001a) hypothesis that the eclipsing binary millisecond pulsar PSR J1740-5340 may be regarded as a recently born binary millisecond pulsar system. Our calculations show that it is possible to build an evolutionary scenario for the binary system with orbital parameters, secondary mass and radius which fit well those for PSR J1740-5340 (D’Amico et al. 2001a,b). In fact, recent photometric and spectroscopic observations by Kałużny et al. (2003) give upper limits for the masses: $M_{\mathrm{ns}}=1.53 \pm 0.19 M_{\odot}$, $M_{\mathrm{sg}}=0.296 \pm 0.034 M_{\odot}$. Also, Orosz \& van Kerkwijk (2003) give limits for the masses: $1.2<M_{\mathrm{ns}} / M_{\odot}<2.4,0.14<$ $M_{\mathrm{sg}} / M_{\odot}<0.38$. These are in good agreement with our predictions. We also suggest that the observed abundances of $\mathrm{C}$, $\mathrm{N}$, O elements in the spectra of the optical companion of the millisecond pulsar can tell us about the evolutionary stage of the secondary. Lack of carbon lines and presence of strong nitrogen lines may tell us that we really have a unique situation involving a pre-He white dwarf + millisecond pulsar system.

Acknowledgements. This work is partly supported through grants 5-P03D-004-21 of the Polish National Committee for Scientific Research and Collaborative Linkage Grant PST.CLG.977383. 


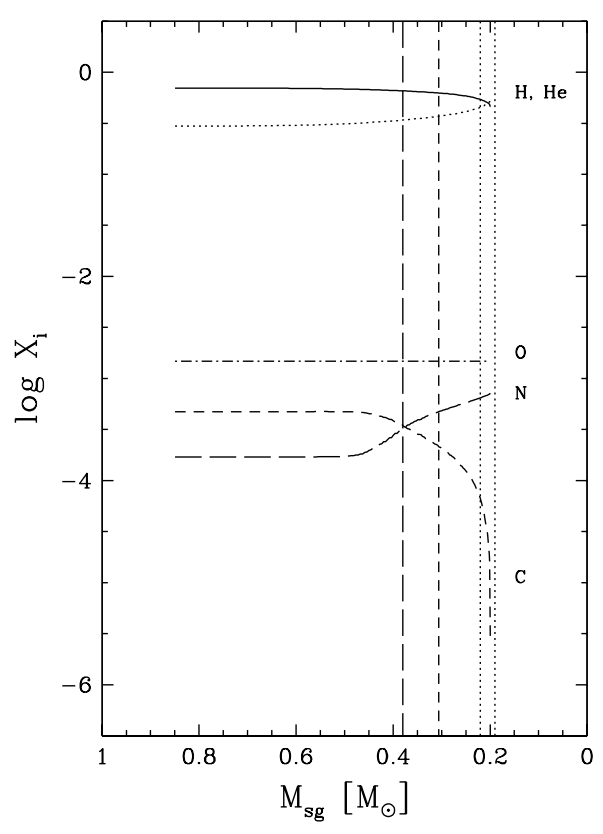

Fig. 4. The evolution of the red giant surface abundances of $\mathrm{H}, \mathrm{He}$, $\mathrm{C}, \mathrm{N}$ and $\mathrm{O}$ as a function of the subgiant mass $M_{\mathrm{sg}} . X_{\mathrm{i}}$ is the mass fraction of $\mathrm{H}, \mathrm{He}, \mathrm{C}, \mathrm{N}, \mathrm{O}$. Note, that the starting values of the abundances are those of a metal poor stars. The vertical dotted lines show the mass range of the secondary star (Ferraro et al. 2001) and the dashed and long-dashed lines are an upper limits for the secondary mass according to Kałużny et al. (2003) and Orosz \& van Kerkwijk (2003), respectively.

EE acknowledge support through Estonian SF grant 4338. We thank anonymous referee for very useful referee opinion.

\section{References}

Burderi, L., Possenti, A., D’Antona, F., et al. 2001, ApJ, 560, L74 Burderi, L., D'Antona, F., \& Burgay, M. 2002, ApJ, 574, 325 Carretta, E., Gratton, R. G., \& Sneden, C. 2000, A\&A, 356, 238

D’Amico, N., Lyne, A. G., Manchester, R. N., Possenti, A., \& Camilo, F. 2001a, ApJ, 548, L171
D’Amico, N., Possenti, A., Manchester, R. N., et al. 2001b, ApJ, 561, L89

Ergma, E., \& Fedorova, A. V. 1992, A\&A, 265, 65

Ergma, E., Sarna, M. J., \& Antipova, J. 1998, MNRAS, 300, 352

Ferraro, F. R., Possenti, A., d'Amico, N., \& Sabbi, E. 2001, ApJ, 561, L93

Fowler, W. A., Caughlan, G. R., \& Zimmerman, B. A. 1967, ARA\&A, 5,525

Fowler, W. A., Caughlan, G. R., \& Zimmerman, B. A. 1975, ARA\&A, 13,69

Grindlay, J. E., Heinke, C. O., Edmonds, P. D., Murray, S. S., \& Cool, A. M. 2001, ApJ, 563, L53

Grindlay, J. E., Camilo, F., Heinke, C. O., et al. 2002 [astro-ph/0208280]

Harpaz, A., \& Rappaport, S. 1991, ApJ, 383, 739

Harris, M. J., Fowler, W. A., Caughlan, G. R., \& Zimmerman, B. A. 1983, ARA\&A, 21, 165

Harris, W. E. 1996, AJ, 112, 1487

Houdashelt, M. L., Bell, R. A., \& Sweigert, A. V. 2000, AJ, 119, 1448

Illarionov, A. F., \& Sunyaev, R. A. 1975, A\&A, 39, 195

Kałużny, J., Ruciński, S. M., \& Thompson, I. 2003, AJ, in press [astro-ph/0209345]

Kudryashov, A. D., \& Ergma, E. V. 1980, Sov. Astron. Lett., 6, 375

Mestel, L. 1968, MNRAS, 138, 359

Mestel, L., \& Spruit, H. 1987, MNRAS, 226, 57

Muslimov, A. G., \& Sarna, M. J. 1993, MNRAS, 262, 164

Muslimov, A. G., \& Sarna, M. J. 1995, ApJ, 448, 289

Orosz, J. A., \& van Kerkwijk, M. H. 2003, A\&A, 397, 237

Podsiadlowski, Ph. 1991, Nature, 350, 136

Pols, O. R., Tout, C. A., Eggleton, P. P., \& Han, Z. 1995, MNRAS, 274, 964

Rakos, K., Schombert, J., Maitzen, H. M., Prugovecki, S., \& Odellaj, A. 2001, AJ, 121, 1974

Taylor, J. M., Grindlay, J. E., Edmonds, P. D., \& Cool, A. M. 2001, ApJ, 553, L169

Tutukov, A. V., Fedorova, A. V., Ergma, E., \& Yungelson, L. R. 1985, SvA, 11, 123

Urpin, V. A., \& Muslimov, A. G. 1992, MNRAS, 256, 261

van den Heuvel, E. P. J. 1977, Ann. NY Acad. Sci., 302, 14

Verbunt, F., \& Zwaan, C. 1981, A\&A, 100, L7 